\title{
Los márgenes del pasado. La producción transdisciplinar del saber arqueológico
}

\section{The limits of the Past. The transdisciplinary production of the Archaeological Knowledge}

\author{
Policarpo Sánchez Yustos \\ policarpo.sy@gmail.com
}

Recibido: 18-06-2013

Aceptado: 24-02-2014

\section{RESUMEN}

Las preguntas filosóficas de la Arqueología han sido respondidas de forma muy diferente por el procesualismo y el postprocesualismo. Para ello, ambas escuelas se apoyan en sistemas filosóficos enfrentados entre los que no existe dialogo. Esta falta de comunicación ha terminado por generar una gran dispersión e inestabilidad epistemológica en el seno de la Arqueología. Así, en este artículo proponemos una construcción participativa y transdisciplinar del Saber arqueológico.

Palabras Clave: Procesualismo, Postprocesualismo, Transdisciplinar, Realismo científico, Relativismo, Arqueosofia.

\begin{abstract}
The current theoretical crisis in Archaeology is caused by the conflict that exists between the philosophical systems of both archeological approaches processualism and postprocessualism. In this paper is proposed a participatory and transdisciplinary construction of the Archaeological knowledge in order to establish the foundations of an alternative theoretical paradigm.
\end{abstract}

Key words: Processualism, Postprocessualism, Transdisciplinary, Scientific realism, Relativism, Archaeosophy. 


\section{Las guerras de la Arqueología}

El desmoronamiento de los metarrelatos teóricos formulados desde la Arqueología ha propiciado que, en las últimas décadas, haya florecido un variopinto catálogo de formas de hacer Arqueología, cada una con sus propios intereses teóricos: from theory to theory of (Hodder 2001: 4). Estas nuevas arqueologías han fundado su identidad gracias a la combinación de un determinado campo de actuación con un cuerpo teórico ad hoc. Se ha generado así un desmigajamiento epistemológico que ha desembocado en un alejamiento progresivo de los interrogantes teóricos de gran calado, a saber: cómo interpretar el pasado y qué tipo de conocimiento genera el arqueólogo. En palabras de Criado Boado, "la teoría arqueológica se ha paralizado y hace tiempo que no ofrece paradigmas" (Criado 2012: 112). Como consecuencia, los márgenes epistemológicos de la reflexión arqueológica siguen confinados entre las estrecheces del procesualimo o entre los dispersos horizontes del postprocesualismo. Si bien es cierto, existen propuestas teóricas recientes que están promoviendo vías alternativas a este dualismo (ej. Webmoor y Witmore 2008; González Ruibal 2009; Olsen 2010; Sánchez Yustos 2010a; González Ruibal et al. 2011; Criado 2012; este trabajo).

Como es sabido, el procesualismo se acerca a las tesis positivistas por lo que hace uso de una epistemología de corte realista, preconizando la existencia de un pasado objetivo independiente de las circunstancias de su descubrimiento. Tal y como pone de relieve Alison Wylie (2002), a la primera ola procesual (ej. Binford 1968; Clarke 1968; Watson et al. 1971) le siguió una notable variedad de enfoques más o menos próximos a lógicas inductivas o deductivas. Sin embargo, todos comparten una filosofía de corte realista que concede una gran importancia a la contrastación de los modelos teóricos. Buena parte de los enfoques procesuales comparten el ideal de hacer de la Arqueología una ciencia al estilo de las Ciencias Naturales, tal y como ha sucedido con la Economía. Por esta razón, la Arqueología procesual ha profundizado en la dimensión económica y ecológica del hombre, desarrollando metodologías cuantitativas centradas en aspectos materiales, lo que ha contribuido a la creación de escenarios descarnados y generalistas. Dicha basculación hacia la dimensión económica es muy notoria en el caso de los estudios paleolíticos (ej. Domínguez Rodrigo 2008; Sánchez Yustos 2012).

La primera reacción al conocimiento esterilizado del procesualismo vino de la mano de grupo de arqueólogos, en su mayoría británicos, que en la década de los ochenta lanzaron una contundente campaña contra la aspiración científica de la Arqueología (ej. Miller y Tilley 1984; Shanks y Tilley 1987, 1992; Tilley 1990). Se pretendía romper con la idea -tan positivade que el pasado puede ser atrapado con las redes del método científico. Para ello se apoyan en una inducción pesimista de la historia de la ciencia, al tiempo que se defiende una ontología relativista y una epistemología subjetivista, que es acompañada de unas estrategias metodológicas cualitativas fundamentadas en la hermenéutica y la dialéctica. En torno a estas coordenadas conviven los múltiples enfoques que se agrupan en lo que se ha dado en llamar Arqueología postprocesual. Sin embargo, la validez de esta etiqueta ha sido contestada por arqueólogos extraoccidentales que han visto en este intento de unidad un acto colonialista (para una actualización del enfoque postcolonialista en Arqueología consultar Gnecco 2012; Haber 2012).

Otra de las líneas maestras con las que se identifica el postprocesualismo es la idea de que el pasado es una construcción polisémica del presente que debe ser entendida en su contexto de producción. Por lo tanto, se considera que todas las interpretaciones son igual de verdaderas y falsas. En otras palabras, el arqueólogo, como sujeto histórico-cultural, es el agente que construye -que no descubre- el pasado. A partir de esta línea argumental se llega a negar la dimensión disciplinar de la Arqueología al compararla con un género literario o una mitología de los orígenes. Es necesario apuntar que esta escuela también debe mucho a la moderna "filosofía de la sospecha" y a la "teoría crítica". Sobre todo, por la preocupación que muestra en clarificar o deconstruir el poso personal, social, económico y político que existe siempre en cualquier interpretación. Asimismo, el postprocesualismo se ha mostrado muy interesado en la des-codificación de los códigos simbólicos que porta la cultura material.

En definitiva, los desencuentros que existen entre el procesualismo y postprocesualismo derivan del hecho de que ambas escuelas se asientan sobre sistemas filosóficos que están en conflicto. De este modo las "guerras de la ciencia" se han instalado en la Arqueología. En la literatura científica, el término Science Wars hace alusión a un episodio de máxima tensión acontecido durante la década de los noventa entre científicos y sociólogos a raíz de un cruce de encendidas publicaciones (Collins y Pinch 1993; Gross y Levitt 1994; Sokal 1996a, 1996b, 1996c; Sokal y Brincmont 1997). Más allá de que esta ofensiva está ligada al engaño pergeñado por el físico Alan Sokal, que vendría 
a remarcar un periodo de pérdida de privilegio y credibilidad por el que pasaba la ciencia más ortodoxa (Martín 1996), en realidad, el germen de estas guerras se encuentra en la separación entre saber natural y saber humanista que fractura la ciencia moderna y que da origen a dos corrientes de pensamiento condenadas a no entenderse: positivismo y antipositivismo, o si se prefiere, realismo y relativismo (Sánchez Yustos 2010a).

\section{La construcción participativa del conocimiento}

Cómo pactar el armisticio y poner fin a las guerras de las ciencias, sin duda alguna, es uno de los grandes retos a los que no sólo se enfrenta la Arqueología del siglo XXI, sino también el conjunto de las Ciencias Sociales. En lo que nos concierne, consideramos que hay dos cuestiones teóricas de gran calado sobre las que los arqueólogos debemos seguir trabajando para ampliar los márgenes de nuestro conocimiento del pasado: equilibrar la balanza objetivo/subjetivo y cartografiar con más precisión la naturaleza del pasado y los problemas que encierra su conocimiento.

Para avanzar en esta dirección, en primer lugar, nos parece conveniente aceptar el poso subjetivo que encierra toda interpretación, determinado por la condición y el contexto del intérprete. Por tanto, cualquier interpretación del pasado está condicionada por el presente. Asumir que la objetividad absoluta no puede ser alcanzada significa que nunca podremos establecer enunciados científicos últimos y que, por ende, debemos conformarnos con alcanzar verdades biodegradables de naturaleza contingente y abiertas a cualquier cambio. Esta concepción pragmática de la verdad no puede resultar un obstáculo que nos impida aproximarnos del modo más fidedigno posible al pasado. Por tal motivo, la principal razón por la que resulta interesante conservar la idea de verdad es que ésta se presente como un horizonte metafísico con ideal regulatorio (Popper 1974: 287).

Por otra parte, resulta evidente la imposibilidad de trazar una frontera nítida entre el presente y el pasado. Entre ambos actúa una relación dialógica y genealógica en la que el pasado hace al presente que a su vez hace al pasado. Este complejo proceso de creación del pasado no agota el hecho de que ocurrió y, por tanto, es posible conocer su realidad, aunque sea de un modo imperfecto.

Este punto de partida nos aleja del frenesí relativista y nos aproxima al "realismo científicocrítico", cuyos orígenes se encuentran en el "racionalismo crítico" de Karl R. Popper (Popper 1956, 1974; Tuomela 1973; Niiniluoto 1999). Según esta posición filosófica, existe una realidad objetiva, independiente del sujeto y regida por leyes naturales. Sin embargo, debemos mantenernos escépticos sobre nuestra capacidad de conocer con certeza (sin error) la estructura categorial y causal de los hechos. La inherente complejidad de la realidad y nuestros imperfectos mecanismos sensoriales y cognitivos impiden su completa aprehensión e irreductibilidad. Dicha complejidad está perfectamente recogida en el mundo cuántico que invalida la existencia de una única realidad, totalmente describible y comprensible en términos de la lógica clásica, lineal, propia de la tradición aristotélica.

Con todo, desde este planteamiento filosófico se reconoce que en ocasiones la realidad es vislumbrada por teorías científicas correctamente establecidas; si bien, su estructura nunca se capta tal y como es en sí misma, sino tal y como nos llega a través de nuestros lenguajes, esquemas conceptuales, instrumentos de medida, etc. Esto supone afirmar que la veracidad o utilidad de las teorías, en último término, es relativa al contexto de descubrimiento, al tiempo que depende del nivel de coherencia que alcanza con el sistema de teorías previamente aceptado.

Por todas estas razones, debemos conformarnos con acercarnos a la realidad de un modo imperfecto y probabilístico. La correspondencia entre algunas formulaciones teóricas abstractas y los datos empíricos, más que a una verdad universal, nos remite a la "inferencia de la causa más probable" (Diéguez Lucena 1998: 19). Nuestros modelos teóricos, provisionales y falibles, son instrumentos que incrementan la capacidad explicativa de la realidad, aunque no deben ser entendidos como meras traducciones. De tal forma, todo conocimiento lleva en sí mismo la marca de la incertidumbre. Aprender a trabajar con esta marca es imprescindible en la medida en que el conocimiento científico sigue siendo falible y su capacidad de progreso depende del error.

Es importante matizar a este respecto que el concepto de realidad que maneja un arqueólogo no es el mismo que el de un físico. Ambos tratan con realidades objetivables aunque de naturaleza muy diferente. Por esta razón, nos parece oportuno distinguir entre realidad continua y realidad discontinua. La primera se refiere a las leyes que gobiernan al mundo físico y que, en principio, tienen una naturaleza constante, mientras que la segunda hace alusión a aquellos hechos socioculturales que sucedieron en el pasado y que no tienen continuidad en el presente. 
El arqueólogo trabaja, pues, con una realidad de naturaleza discontinua que alude a hechos relacionados con las diferentes formas $\mathrm{y}$ expresiones que ha adoptado el comportamiento humano a lo largo del tiempo. Pero esta falta de regularidad y uniformidad dificulta enormemente al arqueólogo la búsqueda y aplicación de leyes y normas en el pasado, lo que supone una restricción del principio ontológico tácito de la investigación científica más ortodoxa (Bunge 2007).

Esta "hipo-realidad" a la que tenemos acceso los arqueólogos y que en gran parte limita nuestras posibilidades de interpretación (Turner 2007), requiere que aceptemos una importante dosis de incertidumbre en cualquiera de nuestras interpretaciones, incluso aunque éstas procedan de alambicados procedimientos científicos. El mayor inconveniente que supone reconocer que no podemos reproducir integral y fidedignamente el pasado, consiste en saber cuál de nuestras interpretaciones se ajusta más a la estructura discontinua de la realidad, que se encuentra fosilizada (de forma incompleta, alterada y, en ciertas ocasiones, deliberadamente codificada y enmascarada) en el registro arqueológico.

Para reforzar los débiles lazos que existen entre el pasado y el presente, siempre que sea posible, es preciso establecer una serie de procesos de evaluación y criterios de demarcación. Esta voluntad de tender cabos firmes hacia el pasado es conveniente que se fundamente en el rigor conceptual, la exactitud, el apoyo en los hechos, la intersubjetividad, la contrastabilidad y revisabilidad, la coherencia o no con otras teorías aceptadas, la capacidad predictiva y la capacidad de progreso.

Esta consistencia la proporciona, sin lugar a duda, el método científico. No obstante, somos conscientes de que su empleo es más apropiado cuando se trabaja con aquellos rasgos del comportamiento humano que están sujetos a una serie de estructuras racionales que gozan de cierta regularidad $y$, en última instancia, pueden ser predichos, contrastados o falsados. Si bien es cierto, las afirmaciones estadísticas y probabilísticas que se obtienen mediante este rígido protocolo de trabajo, con frecuencia son demasiado impersonales y nos sitúan ante un pasado un tanto elemental y excesivamente centrado en aspectos materiales y económicos.

La riqueza y complejidad del comportamiento humano hace que muchas de sus expresiones no puedan ser verificadas en términos de cantidad, intensidad y frecuencia. Por ejemplo, los códigos fenomenológicos que encierra la cultura material difícilmente pueden desvelarse mediante una metodología científica amparada en la teoría de la correspondencia en la que es imprescindible el control experimental. En estos casos de "ciencia postnormal" en los que existe un alto nivel de incertidumbre (Ravetz y Funtowicz 2000), es necesario apelar a una racionalidad argumentativa que fomente la construcción de teorías cuya coherencia y consistencia se identifique con la representación lógica de su organización interna y cuya evaluación intersubjetiva resulte de los criterios marcados por una comunidad interpretativa.

Así las cosas, no podemos afirmar que exista una filosofía de la ciencia capaz de atrapar en sus redes las múltiples aristas que esconde el pasado. De esta forma, y con voluntad de poner de relieve la dimensión procesual y pragmática que encierra el conocimiento, consideramos ineludible el diseño de estrategias de investigación flexibles, cuyo éxito dependa de la capacidad de sus elementos metafísicos para originar explicaciones satisfactorias, coherentes y eficaces.

En gran medida, esta última cuestión depende del empleo de estrategias metodológicas complejas que aborden problemas genuinos sin caer en la tentación de un determinismo racionalizador, hermenéutico o disciplinar que pretenda comprender y explicar cualquier fenómeno a partir de una misma fórmula. Los problemas a tratar y las preguntas a responder, junto con el tipo de registro arqueológico que se estudie, marcan el camino conceptual y metodológico a seguir en cada ocasión, eligiendo así entre el variado abanico de diferentes metodologías que todo investigador tiene al alcance de la mano.

Los ideales regulatorios que acabamos de introducir con intención de alcanzar un punto de unión entre el positivismo y el antipositivismo, se aproxima a la heurística que ofrece la filosofía de la ciencia postpositivista que según Alison Wiley: "are much more likely to engage the questions that arise in archaeological practice than were their antecedents" (2002: 114). El postpositivismo, al igual que el "pensamiento complejo" de Edgar Morin (expuesto con profuso detalle en los diferentes volúmenes de El Método), se muestra interesado en articular la inherente complejidad que gobierna la realidad social y, además, comparte la creencia de que la estimulación dialéctica, crítica y reflexiva suscita la expansión del conocimiento (Fischer 1998). Por otro lado, las aproximaciones sistémicas de las que se nutre el pensamiento complejo favorecen el abandono del supuesto de que la realidad puede ser descrita, analizada y controlada en términos simples, correlacionados a través de la lógica lineal clásica. 
De esta forma, apostamos por una construcción participativa del conocimiento del pasado entre comunidades interpretativas diferentes. Es decir, subscribimos la idea del diálogo epistemológico como estrategia metodológica para acercarnos al pasado tal y como algunos arqueólogos están proponiendo (ej. González Ruibal 2009; Sánchez Yustos 2010b; Criado Boado 2012). Esta idea tan moriniana de ver los antagonismos como complementarios, indudablemente, estimula nuestra capacidad de responder a un espectro de preguntas cada vez mayor y, así, expandir los márgenes de nuestro conocimiento del pasado. Ahora bien, el éxito de este modelo de investigación depende del grado de complejidad y dinamismo que introduzcamos en nuestros enfoques teóricos y metodológicos a la hora de abordar cuestiones particulares.

Nuestra propuesta a favor del dialogo epistemológico como estrategia metodológica y, por tanto, punto de partida para poner fin a las guerras de la arqueología y seguir profundizando en cuestiones teóricas de gran calado, que nos permitan tener un visión más integral y compleja del pasado, no consiste en avanzar hacia una interpretación unificada y monolítica del mismo. Nada más lejos de nuestra intención. Al contrario, nos sumamos aquellos autores que se muestran partidarios del enfoque transdisciplinar (ej. Nicolescu 1996; Gibons et al. 1997; Villar 1997; Morin 2009).

\section{El conocimiento transdisciplinar}

Desde hace ya algunas décadas, tanto a nivel teórico como práctico, se están dando pasos que, desde diferentes direcciones, se alejan progresivamente del determinismo disciplinar que encorseta la producción del conocimiento del pasado en Arqueología. Por ejemplo, el eclecticismo que preside la actual producción arqueológica contiene una importante dosis de transdisciplinaridad y también de aquellas cuestiones más metafísicas relacionadas con la empresa de crear la unidad en el seno de la diversidad. Este último punto ya está siendo abordado desde vías alternativas, como comentábamos al principio del texto, con la intención de liquidar las divergencias y los dualismos cartesianos imperantes en la disciplina. Por otro lado, cada vez más, el pasado se está construyendo gracias al intercambio y cooperación que la Arqueología establece con otras disciplinas.

Sobre esta última cuestión, es importante dejar claro que la transdisciplinaridad no se limita a la investigación cooperativa o a la integración disciplinaria en torno a un tema de investigación, pues este modelo de construcción del conocimiento se denomina interdisciplinar o multidisciplinar. La transdisciplinaridad se diferencia en que se ocupa de combatir los principales peligros de la disciplinaridad, tales como la parcelación, el aislamiento y la simplificación del conocimiento; así como la incomunicación con sus contrarios que genera el saber-poder disciplinario.

La visión transdisciplinar persigue un conocimiento global, integral y complejo. Une conocimientos en vez de separarlos, al tiempo que propone considerar una realidad multidimensional que reemplaza la realidad unidimensional del pensamiento clásico. Representa, por tanto, un claro desafío a la lógica binaria y lineal de la tradición aristotélica. Su intención es superar la parcelación y fragmentación del conocimiento. Disuelve las fronteras disciplinares para, desde una racionalidad abierta y una lógica dialéctica, aproximarse de manera más compleja a la realidad, atendiendo a la multidimensionalidad de los fenómenos a estudiar y promoviendo el dialogo epistemológico y metodológico. Es decir, busca estimular la comunicación y reconciliación de aparatos teórico-metodológicos antagónicos: contraria sunt complementa. Su gran reto es recomponer la realidad fragmentada por la disciplinarización del saber, creando un corpus de conocimiento que transcienda cualquier disciplina. Pero la transdisciplinaridad no surge para eliminar las disciplinas, sino para poner fin a la pretensión exagerada que supone que desde la perspectiva de una disciplina o episteme aislada se puede aportar un conocimiento totalizador sobre la realidad.

Al abandonar el modelo dual, positivista y vectorial de la realidad, la transdisciplinaridad se adentra en un modelo dialéctico, emergente, más incierto y complejo. Su matriz filosófica postpositiva niega todo sistema cerrado de pensamiento y se introduce en el paradigma de la complejidad y, por ende, en el paradigma sistémico. Así, asume la imposibilidad de una teoría completa cerrada sobre sí misma. Cada teoría en un nivel dado de realidad, es una teoría transitoria, ya que inevitablemente lleva a descubrir nuevas contradicciones situadas en nuevos niveles de realidad. Este proceso puede continuar indefinidamente sin nunca alcanzar la construcción de una teoría unificada completa. El conocimiento es, entonces, una estructura abierta, mientras que la realidad es definida como multidimensional y multirreferencial.

Una construcción transdisciplinaria y compleja del conocimiento del pasado se caracteriza por estar centrada en los fenómenos más que en 
la disciplina, en la incertidumbre más que en la certeza, en el acontecimiento más que en la variable, y en la ruptura y la emergencia más que en la regularidad de los hechos planteados de forma determinista. El pasado, examinado desde el pensamiento transdisciplinar, es hechura de la multiperspectividad al patrocinar el estudio integral de una realidad pretérita armonizando horizontes interpretativos antagónicos. En otras palabras, no opone al procesualismo el postprocesualismo o viceversa; al contrario, los considera como racionalidades complementarias y válidas para el conocimiento de dicha realidad.

La perspectiva paisajista en Arqueología se ha convertido, de manera involuntaria, en un modelo de referencia de investigación transdisciplinar y complejo (Sánchez Yustos 2010b). A nuestro entender, se trata del mejor ejemplo arqueológico de construcción del conocimiento del pasado elaborado a partir de diferentes comunidades interpretativas, ya que la interacción de epistemologías y métodos antagónicos ha estimulado la comprensión multidimensional de dicho fenómeno.

En torno al concepto de Paisaje el procesualismo y el postprocesualismo han cruzado sus respectivas filosofías de la ciencia dando como resultado un estudio integral de las diferentes dimensiones que ofrece el Paisaje, convertido a éste en una fenómeno paradigmático dentro del campo de la Arqueología. Al hablar del Paisaje como un fenómeno paradigmático nos referimos a cómo, en torno a esta categoría de análisis, se están diseñando programas de investigación que combinan diferentes enfoques teórico-metodológicos que, de forma conjunta, proporcionan explicaciones holísticas, más comprensivas, de cómo las personas y las sociedades del pasado han dado forma al Espacio. Fundamentar la construcción del conocimiento del pasado sobre la complejidad que surge de armonizar diferentes epistemologías y estrategias de trabajo en torno a este fenómeno, por un lado, ha permitido contrastar intersubjetividades con datos objetivos (y viceversa) y, por el otro, desarrollar un modelo de investigación que no está presidido tiránicamente por la ciencia positiva o por la oscuridad del relativismo.

\section{La encrucijada}

La producción transdisciplinar del conocimiento del pasado en los términos propuestos, a nuestro juicio, debe acompañarse de una reformulación del propio concepto de Arqueología. Su etimología, por un lado nos remite a archaia, "las cosas viejas"; y por el otro, logia habla de estudio, tratado y recta razón. Por tanto, Arqueología hace referencia al "estudio de lo antiguo basado en la recta razón". El determinismo racionalizador que encierra esta definición choca frontalmente con nuestra reclamación de un saber integral del pasado.

Por tal motivo, proponemos el término de «Arqueosofía», que etimológicamente significa "el Conocimiento o Saber del pasado", y hace referencia a Saber como objetivo final. $\mathrm{Su}$ eficacia metodológica resulta de deslocalizar disciplinarmente la producción del conocimiento del pasado y armonizar una serie de teorías y métodos que, aunque sus discursos puedan resultar antagónicos, son el medio para acceder a ese Saber.

Este nuevo concepto, más que una nueva disciplina o meta-disciplina, en realidad, es un modo diferente de acercarse al conocimiento arqueológico, más complejo y holístico. Por otro lado, su "omnivorismo" intelectual permite fusionar en su seno cualquier racionalidad lógica y coherente $\mathrm{y}$, en última instancia, construir un Saber acumulativo, complejo y dinámico. Es importante puntualizar que Arqueosofía no es un término designado al almacenaje de toda discusión filosófica sobre la naturaleza del conocimiento arqueológico y su identidad como disciplina, aunque ahí se encuentra su origen. En rigor, hace alusión a un marco epistemológico multivocal que hace del diálogo, la participación, la cooperación, el encuentro y el intercambio, piezas fundamentales de una estrategia cognoscitiva consignada a la expansión de los márgenes de nuestro Saber-del-pasado.

La introducción de este concepto y de la reflexión teórica que lleva aparejada nos conduce directamente a la encrucijada en la que Criado Boado sitúa a la Arqueología: "¿Se trata de un saber o un servicio, una ciencia o una tecnología?, que no es lo mismo que la falsa disyuntiva entre investigación y gestión" (Criado 2012: 114). En su opinión, la patrimonialización del registro arqueológico ha supuesto el cambio más drástico que la Arqueología ha experimentado en los últimos cien años, propiciando su fragmentación, por cuanto su constitución como actividad liberal, su introducción en el mercado y el surgimiento y consolidación de un sector profesional nuevo, ha propiciado la disolución de la investigación como práctica rectora de la Arqueología (Criado 2012: 114). Otro elemento de validación de esta disyuntiva entre la dimensión teórica y práctica es que "frente al carácter hipotético que tiene el conocimiento arqueológico sobre el pasado desvanecido, la gestión del patrimonio maneja certezas contrastables" (Criado 2012: 122). En definitiva, para Criado Boado "la Arqueología tal 
y como la hemos comprendido hasta la fecha no se puede mantener" (Criado 2012: 114).

Llegados a esta encrucijada puede resultar de cierta utilidad asimilar el concepto de Arqueología al de Saber-hacer y el de Arqueosofía al de SaberConocer. Dicho de otro modo, "la finalidad debe ser reconvertir la Arqueología en una tecnología de gestión del patrimonio arqueológico" (Criado 2012: 119), mientras que la Arqueosofía se convierte en la construcción científica del conocimiento del pasado basada en el registro material recuperado mediante dicha práctica. Ambos conceptos mantienen una relación dialógica por cuanto la combinación indisoluble entre sabiduría y aplicación requiere de la partición y diálogo de diferentes agentes, disciplinas, epistemes y técnicas. La dualidad Arqueosofía-Arqueología se convierte, por tanto, en un sistema teórico-práctico transdisciplinar y complejo de conocimiento y gestión del patrimonio arqueológico.

\section{REFERENCIAS BIBLIOGRÁFICAS}

BINFORD, L.R. (1968): Archaeological perspectives. New perspective in archaeology (S.R. Binford y L.R. Binford eds.), Aldine, Chicago: 5-32.

Bunge, M. (2007): A la caza de la realidad. La controversia del realismo. Gedisa, Barcelona.

Clarke, D.L. (1968): Analytical archaeology. Methuen, London.

Collins, H.; PInch, T. (1993): The golem: What everyone should know about science. Cambridge University Press, Cambridge.

Criado Boado, F. (2012): Arqueológicas: la razón perdida. La construcción de la inteligencia arqueológica. Bellaterra, Barcelona.

Diéguez Lucena, A.J. (1998): Los compromisos del realismo científico. Filosofía actual de la ciencia, 3: 145-173.

Domínguez Rodrigo, M. (2008): Arqueología neo-procesual: alive and kicking. Algunas reflexiones desde el Paleolítico. Complutum, 19(1): 195-204.

FISCHER, F. (1998): Beyond empiricism: policy inquiry in postpositivist perspective. Policy Studies Journal, 26(1): 129-146.

FunTOWICZ S.O.; RAVETZ, J.R. (1990): Uncertainty and quality in science for policy. Kluwer, Dordrecht.

Gibbons, M.; Limoges, C.; Nowotny, H.; Schwartzman, S.; Scott, P. y Trow, M. (1997): La nueva producción del conocimiento. La dinámica de la ciencia y la investigación en las sociedades contemporáneas. Pomares, Barcelona.

Gnecco, A. (2012): Arqueología multicultural. Notas intempestivas. Complutum, 23(2): 93-102.

GonzÁlez Ruibal, A. (2009): Sobre los límites del constructivismo social y las posibilidades del concepto de verdad. Complutum, 20(1): 175-196.

González Ruibal, A.; Hernando, A.; Politis, G. (2011): Ontology of the self and material culture: arrow-making among the Awá hunter-gatherers (Brasil). Journal of Anthropological Archaeology, 30: 1-16

Gross, P.R.; LevitT, N. (1994): Higher superstition: the academic left and its quarrels with science. Johns Hopkins University Press, Baltimore.

Haber, A. (2012): Tiempo de carnaval. Colonialidad de la arqueología y semiopraxis de la serpiente. Complutum, 23(2): 117-126.

HodDer, I. (2001): Introduction: A review of contemporary theoretical debates in archaeology. Archaeological theory today (I. Hodder ed.). Polity, Cambridge.

Martin, B. (1996): Social construction of an "attack on science". Social Studies of Science, 26: 161-173

Miller, D.; Tiller, C. (1984): Ideology, Power and Prehistory. Cambridge University Press. Cambridge.

Morin, E. (2009): Introducción al Pensamiento Complejo. Gedisa, Madrid.

Ninilluoto, I. (1999): Critical Scientific Realism. Oxford University Press. Oxford.

Nicolescu, B. (1996): La transdisciplinarité manifeste. Le Rocher, París.

Olson, D.R. (2010): In defense of Things. Archaeology and the Archaeology of Objects. Altamira Press, Maryland.

Popper, K.R. (1956): Realism and the aim of the science. Bartley III, London.

Popper, K.R. (1974): Conocimiento objetivo. Tecnos, Madrid.

Ravetz, J.R.; Funtowicz, S.O. (2000): Ciencia posnormal. Ciencia con la gente. Icaria, Barcelona. 
SÁnchez Yustos, P. (2010a): Arqueología de la Modernidad. Revista de Filosofía, 34(2): 115-137.

SÁnchez Yustos, P. (2010b): Las dimensiones del Paisaje en Arqueología. Munibe, 61: 157-173.

SÁNChez Yustos, P. (2012): Crítica a la cultura fósil. La estructura económica como unidad de análisis del cambio cultural paleolítico. Complutum, 23(1): 27-40.

Shanks, M.; Tilley, C. (1987): Social theory and archaeology. Polity. Oxford.

Shanks, M.; Tilley, C. (1992): Re-constructing Archaeology: theory and practice. Routledge, London.

Shanks, M. (2001): Culture/Archaeology: The dispersion of a discipline and its objects. Archaeological theory today (I. Hodder ed.). Polity, Cambridge.

SoKal, A. (1996a): Transgressing the boundaries: Toward a transformative hermeneutics of Quantum Gravity. Social Test, 46/47: 217-252.

SoKal, A. (1996b): A physicist experiments with cultural studies. Lingua Franca, 6: 62-62.

SoKAl, A. (1996c): Transgressing the boundaries: An afterword. Dissent, 43: 93-99.

SoKAl, A.; Bricmont, J. (1997): Impostures intellectuelles. Odile Jacob, Paris.

Tilley, C. (1990): Reading material culture. Structuralism. Hermeneutics and post-structuralism. Blackwell, Oxford.

Toumela, R. (1973): Theoretical concepts. Springer. New York.

Turner, D. (2007): Making Prehistory. Historical science and scientific realism debate. Cambridge University Press, Cambridge.

VILlar, S. (1997): La nueva racionalidad. Comprender la complejidad con métodos transdisciplinarios. Kairós. Barcelona.

Watson, P.J.; Leblanc, S.A.; Redman, C. (1971): El método cientifico en arqueología. Alianza. Madrid.

Webmoor, T.; Witmore, C.. (2008): Things Are us! A Commentary on Human/things Relations under the banner of a 'Social' Archaeology. Norwegian Archaeological Review, 41(1): 53-70.

Wylie, A. (2002): Thinking from things. Essays in the philosophy of archaeology. University of California Press, Berkeley. 